\title{
Especial
}

\section{Actividades feminizadas y el amianto: los hallazgos "casuales"}

\section{Jobs developed by women and asbestos: The "accidental" findings}

\section{María Fernanda González Gómez}

\author{
Servicio de Salud Laboral. Dirección General de Ordenación e Inspección. Consejería de Sanidad de la \\ Comunidad de Madrid. Madrid.
}

\section{Recibido: 01-06-11}

Aceptado: 26-05-11

\author{
Correspondencia \\ María Fernanda González Gómez \\ P. ${ }^{\circ}$ de Recoletos n. ${ }^{\circ}$ 14, 4. ${ }^{a}$ planta \\ 28001 Madrid. España \\ Tfno: 914269347 \\ e-mail: mfernanda.gonzalez@salud.madrid.org
}

Resumen

La exposición a fibras de amianto constituye un riesgo para la salud de los y las trabajadoras, que puede verse afectada muchos años después del contacto con esta sustancia. En cumplimiento de la normativa europea y nacional, se ha puesto en marcha un programa de vigilancia de la salud de estas personas en la Comunidad de Madrid. Como paso previo se ha reconstruido la cohorte de sujetos expuestos a los que hacer dicho seguimiento. Dicha cohorte está constituida mayoritariamente por hombres (casi el 96,5\%), que son los que en mayor número realizaban actividades de fabricación de productos y piezas con amianto. Sin embargo, el descubrir una mujer afectada por amianto, trabajadora de una empresa sin exposición conocida ni declarada, puso de manifiesto que existen otra serie de actividades relacionadas con dichas fibras, tradicionalmente realizadas por mujeres, y que no habían sido consideradas como de riesgo de amianto, por estar invisibilizadas. Introducir la perspectiva de género en las actividades y programas de las instituciones mejorará la calidad de los mismos, y permitirá la equidad real en el ejercicio del derecho de hombres y mujeres en el ámbito de la salud laboral.

Med Segur Trab (Internet) 2011; 57 (223) 106-110

Palabras clave: amianto, enfermedad profesional, género.

\begin{abstract}
The exposure to asbestos fibers is a real risk for workers' health, that can be affected many years after the contact with this substance. In compliance with current European and National legislation, a specific program has been developed in Madrid, to follow these people's health. Firstly, a cohort of exposed people whose health must be followed has been created. This cohort is mostly comprised of males (96,5 \%), who worked manufacturing products containing asbestos. However, having discovered a woman who had worked for a company with no declared exposure, ill from a disease related to asbestos, has revealed that there are other kinds of occupational activities in contact with these fibers, never having been considered as activities at risk because, since they were developed by women, they were invisible. The introduction of gender perspective in institutions' activities and programs, will improve their quality and it will favour the real equity in the exercise of workers rights for both, men and women.
\end{abstract}

Med Segur Trab (Internet) 2011; 57 (223) 106-110

Keywords: asbestos, occupational disease, gender. 


\section{INTRODUCCIÓN}

Las palabras "amianto" o "asbesto" se utilizan para nombrar un amplio grupo de sustancias fibrosas formadas por silicatos. Las fibras de amianto tienen unas características físicas que le convierten en un material especialmente atractivo para diferentes industrias y actividades (incombustibilidad, aislamiento térmico, acústico y eléctrico, resistencia a la abrasión...). En España, su uso masivo tuvo lugar entre los años 1960 y 1984, y la fabricación y comercialización de la última variedad de amianto permitida en el país —el crisotilo- fue prohibida sólo hace 9 años (junio de 2002).

La exposición a las fibras de amianto supone un riesgo importante para la salud, con efectos a largo plazo, produciendo, entre otros: fibrosis pulmonar difusa y progresiva (asbestosis), fibrosis pleural o pericárdica con restricción pulmonar o cardiaca, cáncer de pulmón o mesotelioma maligno (pleural o peritoneal); todas ellas incluidas en el listado de Enfermedades Profesionales (si existe exposición laboral previa), aprobado por el Real Decreto 1299/2006. Pueden aparecer también otras lesiones derivadas de la exposición tales como placas pleurales, atelectasias redondas u otras que, en sí mismas, no son susceptibles de ser reconocidas como enfermedad profesional si no se acompañan de repercusión funcional. Dado el largo periodo de latencia que presentan estas patologías desde la exposición a las fibras (puede llegar a ser de hasta 30 y más años para alguna de ellas), muchos de los nuevos casos detectados se encuentran ya jubilados (o hace muchos años que se separaron de la empresa en la que estuvieron expuestos al amianto), y son diagnosticados por servicios de salud no relacionados con la medicina del trabajo, por lo que es muy frecuente que no se investigue la posible exposición laboral al asbesto.

Con el fin de subsanar este hecho, y en cumplimiento de la normativa vigente, el Servicio de Salud Laboral de la Consejería de Sanidad de la Comunidad de Madrid trabaja desde el año 2003 en la localización de personas que estuvieron o están expuestas en su medio de trabajo al amianto, en el marco del Programa Integral de vigilancia de la salud de los trabajadores expuestos a amianto, programa aprobado por la Comisión Nacional de Salud en el Trabajo y por el Consejo Interterritorial del Sistema Nacional de Salud en el año 2003.

Hasta ahora el listado de empresas en las que se dio o en las que actualmente se da exposición al amianto incluye aquellas en las que se fabricaban productos de fibrocemento, frenos, embragues, juntas, vehículos, barcos, y también aquellas relacionadas con la construcción, demolición y gestión de residuos contaminantes. Asimismo están en el listado otras empresas que prestan diversos servicios, como mantenimiento de instalaciones de distribución de agua, de refrigeración, calefacción, etc. Desgraciadamente, es casi imposible localizar a otros trabajadores (fontaneros, albañiles, mecánicos, etc.), que en el desarrollo de su actividad han tenido exposición a amianto pero sin conocerla, y que, por su condición de autónomos no cotizaban y por tanto no tenían derecho al reconocimiento de contingencia profesional en el caso de presentar patología discapacitante relacionada con el amianto.

La población trabajadora expuesta que constituye nuestra cohorte está mayoritariamente conformada por hombres $(96,5 \%)$, y, en las empresas en las que se manejaban las fibras de amianto (en las que la prevención era prácticamente inexistente), eran éstos los que estaban más expuestos dada la segregación de ocupaciones entre ambos sexos (en cuanto horas y trabajo en el ambiente pulvígeno). No obstante fueron consideradas también como expuestas, además de las personas cuya ocupación les ubicaba en la fuente de la exposición, aquellas empleadas en tareas administrativas o de hostelería, dada la profusa presencia de fibras de amianto en todas las instalaciones, incluyendo así a las mujeres que sufrieron la exposición en dichas actividades (actividades realizadas fundamentalmente por trabajadoras). Quedaron fuera del registro muchas mujeres expuestas en su quehacer cotidiano, consistente en lavar la ropa de trabajo de sus esposos (no hubo cotización por ello, y por tanto no hay opción al reconocimiento de enfermedad profesional en el caso de presentarse lesiones relacionadas). También 
quedaron fuera aquellas mujeres que informalmente trabajaban para algunas de estas empresas en la reparación de sacos, uniformes de trabajo, etc, y que sí cobraban por ello aunque nunca mediase contrato escrito.

Sin embargo, en algunas ocasiones aparece la oportunidad de incorporar a la cohorte a otras personas expuestas a estas fibras, y cuya tarea ni siquiera se imaginó como actividad con riesgo de exposición al amianto: no es infrecuente descubrir que, en actividades "feminizadas", los riesgos laborales no han sido valorados adecuada o suficientemente. En el caso que vamos a describir han jugado un papel definitivo algunos factores concluyentes que van más allá de la sistemática de nuestro programa para incorporar personas expuestas en el medio laboral, y que tienen más que ver con el interés de las personas implicadas y con el trabajo que se está haciendo para introducir la perspectiva de género en las actividades del servicio de Salud Laboral, (entre ellas, la formación a las y los futuros especialistas en Medicina del Trabajo).

\section{ASBESTOSIS EN UNA TRABAJADORA EN UNA ACTIVIDAD NO CONOCIDA COMO RIESGO}

El origen de este artículo está en la atención que recibe en urgencias de un hospital madrileño una mujer de sesenta y cinco años que acude por una insuficiencia respiratoria aguda. Esta mujer es atendida en su jornada de guardia por una médica residente de Medicina del Trabajo que había rotado por este Servicio de Salud Laboral, y a la que muestra informes previos que relacionan su patología respiratoria de base con asma o con patología intersticial no filiada, y en los que se plantea la posible exposición a amianto, por algunas imágenes de placas pleurales y patrón reticular parenquimatoso.

Esta residente se interesa por su actividad profesional previa y, por primera vez en la historia de su enfermedad, ante la sospecha de la exposición laboral al amianto, se actúa en consecuencia con esta mujer: notifica el caso a este servicio de Salud Laboral, desde donde se inician las gestiones pertinentes para aclarar si efectivamente hubo o no dicha exposición. Aunque no todos los profesionales tienen presente la historia laboral de sus pacientes, cuando a uno de ellos se le detecta una patología respiratoria relacionada con el amianto, es habitual que se le recomiende iniciar la gestión para el reconocimiento de la EP. Por otro lado, aún si no se existe dicha recomendación, muchos son los trabajadores conocedores de este derecho, dada la cercanía de compañeros afectados que disfrutan del mismo. Sin embargo, esta trabajadora no conocía su exposición laboral al amianto puesto que, habiendo trabajado en una empresa pequeña cuya principal actividad no era la producción de productos que contuviesen esta fibra, no disponía de ninguna otra información al respecto (ni siquiera procedente de sus compañeros/as), que aquella que en ese momento recibe en el servicio sanitario de urgencias.

Desde el Servicio de Salud Laboral nos pusimos en contacto con la interesada, con el objeto de investigar la posible exposición laboral al asbesto, solicitándole la historia de vida laboral: a la vista de la misma pudimos confirmar que había trabajado en una empresa dedicada a la venta, preparación, clasificación, reparación y comprobación de sacos de diferentes materiales. Según relató la paciente, muchos de estos sacos estaban impregnados de un polvo gris que "decían que era amianto", y su actividad consistía en coserlos - los rotos - y prepararlos para nuevos usos. Se trataba de una empresa familiar con pocos trabajadores, y esta actividad ocupaba gran parte de las tareas a realizar.

Con el fin de confirmar que efectivamente parte de los sacos manipulados estaban contaminados con amianto, se solicitó formalmente a la administración laboral autonómica que investigase este punto. Finalmente, tras visitar la empresa y entrevistarse con su propietario y gerente, fue confirmado el testimonio de la mujer: Entre los años 60 y 1972-1973, esta empresa prestaba sus servicios, entre otras, a una industria importante en la región dedicada a la producción de productos de fibrocemento, manteniendo los stocks de sacos en los que se almacenaba el asbesto. Según la investigación, las tareas con 
los sacos que habían contenido amianto consistían en el sacudido de los sacos para su posterior clasificación y venta (estos sacos no eran cosidos en el caso de estar rotos o deteriorados). Se consiguió, pues, probar la exposición laboral al amianto más de seis años después de la aparición de los efectos del mismo en la salud de esta trabajadora.

Tras ponernos en contacto con ella, acudió a nosotros su hermana, que había también trabajado en la misma empresa, y que igualmente presentaba síntomas respiratorios. Ambas aportaron sus informes médicos, y con éstos y el informe emitido sobre la exposición en la empresa, se iniciaron los trámites ante el INSS para solicitar el reconocimiento de la contingencia profesional. Recientemente el equipo de valoración de incapacidades ha reconocido la dolencia de ambas como enfermedad profesional, lo que conllevará un derecho económico que, aunque no contribuya a tratar su patología, sí les facilitará la mejora de las condiciones en que viven debidas, en parte, a la disfunción respiratoria que sufren por su exposición al asbesto.

\section{DISCUSIÓN}

Todos estos hechos ponen de manifiesto, por un lado, que la actitud proactiva de las instituciones en la búsqueda de personas expuestas a amianto en el medio laboral es indispensable para mejorar la calidad de los registros, y para contar con una cohorte que incluya el mayor número de sujetos expuestos, que facilite el seguimiento de su salud y el reconocimiento como Enfermedad Profesional de aquellas patologías relacionadas con la exposición. Asimismo este caso muestra que una adecuada coordinación y colaboración entre instituciones es imprescindible para afrontar de forma exitosa un programa de las características del que nos ocupa. La adecuada gestión de estas dos trabajadoras afectadas hubiera sido del todo imposible sin la colaboración con el Instituto Regional de Seguridad y Salud en el Trabajo y la coordinación con la Dirección Provincial de INSS, en el marco del convenio firmado entre esta instancia y la Consejería de Sanidad de nuestra comunidad autónoma.

Por último, estos hechos desvelan el sesgo de género subyacente en la mayoría de las actuaciones de instancias públicas y privadas, y en el personal técnico que trabajamos en ellas. Se conocen actividades y ocupaciones en las que pudo haber exposición (en las que participaron mayoritariamente hombres), y de las que se sabe que desgraciadamente no se podrán obtener más datos, —-debido sobre todo a la gran dispersión de ocupaciones de la población trabajadora expuesta, y a la gran bolsa de trabajadores autónomos que no tienen la contingencia profesional cubierta-, y se cuenta de antemano con dicho handicap. Sin embargo, en ningún momento se habían planteado actividades como la que articula este escrito, y a pesar de que se trata de una tarea necesaria para casi cualquier industria, y que seguramente habrán llevado a cabo múltiples empresas en todo el país - y cómo no, en todo el mundo-, no había sido contemplada como actividad de riesgo. Así pues, seguramente estamos hablando de un número considerable de personas expuestas, oculto tras las cifras de las grandes industrias. Y dado que son las mujeres las que mayoritariamente han llevado a cabo este tipo de actividades de fondo, estaríamos dejando a un lado, de nuevo, la prevención, la vigilancia y el reconocimiento del daño de estas trabajadoras, sujetos — sin embargo- de pleno derecho.

Esta actividad y su exposición al amianto ha permanecido invisible incluso a los ojos de las instancias encargadas de la creación de registros de personal expuesto; se trataba de una actividad principalmente feminizada, y como tal había quedado ella y los riesgos que conllevaba en un segundo plano, oculta: una vez más la invisibilidad de las mujeres en el ámbito laboral tiene efectos negativos sobre su salud y sobre el ejercicio real de sus derechos.

Sin embargo, esta presencia femenina en determinadas actividades de riesgo queda manifiesta cuando existe voluntad de investigar, aún someramente, los casos. Introducir, pues, la perspectiva de género en las actuaciones de las administraciones sanitarias y 
laborales constituye una herramienta necesaria y muy válida para garantizar la calidad en sus actuaciones, y para promover la equidad en la población trabajadora.

El hecho de que exista esta voluntad en las administraciones públicas es esperanzador, y en un futuro que deseamos cercano estos hallazgos no serán fruto de una serie de casualidades azarosas y voluntades personales, sino más bien de una estrategia bien diseñada y del uso sistemático de indicadores de calidad con perspectiva de género.

\section{REFERENCIAS BIBLIOGRÁFICAS}

1. Comunidad de Madrid. Programa para la vigilancia de los trabajadores que han estado expuestos a amianto en la Comunidad de Madrid. Consejería de Sanidad. Comunidad de Madrid. Madrid, 2008. Disponible en: http://www.madrid.org/cs/Satellite?cid=1142333143941\&language=es\&pageid=11423331 41667\&pagename=PortalSalud\%2FPTSA_Generico_FA\%2FPTSA_pintarGenerico\&vest=1156329914017

2. Directiva 83/477/CEE del Consejo sobre la protección de los trabajadores contra los riesgos relacionados con la exposición al amianto durante el trabajo. Diario Oficial de la Unión Europea n. ${ }^{\circ}$ L 263 de 24 de septiembre de 1983 .

3. Directiva 91/382/CEE del Consejo por la que se modifica la Directiva 83/477/CEE del Consejo sobre la protección de los trabajadores contra los riesgos relacionados con la exposición al amianto durante el trabajo. Diario Oficial de la Unión Europea n. ${ }^{\circ}$ L 206 de 29 de julio de 1991

4. Directiva 1999/77/CE de la Comisión por la que se adapta al progreso técnico por sexta vez el anexo I de la Directiva 76/769/CEE del Consejo relativa a la aproximación de las disposiciones legales, reglamentarias y administrativas de los Estados miembros que limitan la comercialización y el uso de determinadas sustancias y preparados peligrosos (amianto). Diario Oficial de la Unión Europea n. ${ }^{\circ}$ L 207 de 6 de agosto de 1999.

5. Directiva 2003/18/CE del Parlamento Europeo y del Consejo, de 27 de marzo de 2003, por la que se modifica la Directiva 83/477/CEE del Consejo sobre la protección de los trabajadores contra los riesgos relacionados con la exposición al amianto durante el trabajo. Diario Oficial de la Unión Europea, 15 de abril 2003 (n. $\left.{ }^{\circ} 20\right)$

6. Ley 31/1995, de 8 de noviembre, de Prevención de Riesgos Laborales. B.O.E. n. ${ }^{\circ} 269$ de 10 de noviembre de 1995.

7. Ministerio de Sanidad y Consumo (MSC). Programa integral de vigilancia de la salud de los trabajadores que han estado expuestos a amianto y protocolo de vigilancia sanitaria específica (revisión 2003). Disponible en: www.msc.es/ciudadanos/saludAmblaboral/docs/protocoloAmianto.pdf.

8. Orden de 31 de octubre de 1984 por la que se aprueba el Reglamento sobre trabajos con riesgo de amianto. B.O.E. n. ${ }^{\circ} 267$ de 7 de noviembre de 1984.

9. Orden de 7 de diciembre de 2001 por la que se modifica el anexo I del Real Decreto 1406/1989, de 10 de noviembre, por el que se imponen limitaciones a la comercialización y al uso de ciertas sustancias y preparados peligrosos. Ministerio de la Presidencia. B.O.E. n. ${ }^{\circ} 299$ del 14 de dicimebre de 2001.

10. Real Decreto 39/1997 de 17 de enero de 1997, por el que se aprueba el Reglamento de los Servicios de Prevención. B.O.E. n. ${ }^{\circ} 27$ de 31 de enero de 1997.

11. Real Decreto 665/1997 sobre la protección de los trabajadores contra los riesgos relacionados con la exposición a agentes cancerígenos durante el trabajo. B.O.E. n. ${ }^{\circ} 124$ de 24 de mayo de 1997.

12. Real Decreto $1124 / 2000$, de 16 de junio por el que se modifica el Real Decreto 665/1997. B.O.E. n. ${ }^{\circ} 145$ del 17 de junio de 2000

13. Real Decreto 396/2006, de 31 de marzo, por el que se establecen las disposiciones mínimas de seguridad y salud aplicables a los trabajos con riesgo de exposición a amianto. B.O.E n. ${ }^{\circ} 86$, de 11 de abril de 2006. B.O.E. n. ${ }^{\circ} 86$ de 11 de abril de 2006

14. Real Decreto $1299 / 2006$, de 10 de noviembre, por el que se aprueba el cuadro de enfermedades profesionales en el sistema de la Seguridad Social y se establecen criterios para su notificación y registro. B.O.E n. ${ }^{\circ}$ 302, de 19 de diciembre de 2006. 